\title{
La polysémie verbale appréhendée par une sémantique cognitive et formelle
}

\author{
Jean-Pierre Desclés ${ }^{1, \mathrm{i}}$ et Zlatka Guentchéva ${ }^{2}$ \\ 1 STIH, Sorbonne Université, 28 rue Serpente, 75006 Paris, France \\ 2 LACITO- UMR7107, CNRS, 7bis, rue Guy Môquet, 94800 Villejuif, France
}

\begin{abstract}
Résumé. L'étude de la polysémie verbale (et prépositionnelle) revient à construire un réseau dont la racine est un invariant sémantique et les nœuds des schèmes. Chaque schème représente une signification sous la forme de relations emboîtées de situations construites à l'aide de primitives sémantiques : types primitifs des différentes entités ; primitives relationnelles telles que le mouvement, le changement, l'effectuation d'une action, le contrôle et la visée téléonomique. Ces primitives sont liées aux activités cognitives de perception et d'action, plus ou moins intentionnelles. L'article traite plus spécialement des verbes polysémiques français, 'arrêter', s'arrêter', 'garer' et 'se garer'.
\end{abstract}

\begin{abstract}
Verbal polysemy apprehended by a cognitive and formal semantics. The study of verbal polysemy (and prepositions) leads to construct a network with at the top a semantic invariant and schemes at different points of the network. A scheme represents a meaning by means of relations formulated with the help of semantic and cognitive primitives: primitive types (basic categorizations) of entities; relational primitives such as to move, to change, to do an action, to control a change or a movement, to aim a final situation. These primitives are related to the cognitive activities of perception and more or less intentional actions. The purpose of the article is a formal representation by means of schemes of different meanings of verbs of French 'arrêter', 's'arrêter', 'garer' and 'se garer'.
\end{abstract}

\section{Introduction}

Des ouvrages comme ceux de Fuchs et Victorri (1996), Kleiber (1999), Soutet, ed. (2005) ou François (2007), proposent différentes approches de la polysémie. Notre propre approche sémantique de la polysémie nous a conduit à étudier la polysémie de verbes comme monter, avancer, toucher, porter, donner (Desclés 2005, 2011a), puis celle de prépositions comme sur, sous, entre... (Desclés 2001, 2004), en comparant ces unités du français avec leurs traductions dans d'autres langues (albanais, bulgare, polonais, russe, anglais, coréen...). Nous reprenons à nouveau ce problème en traitant particulièrement les verbes arrêter et s'arrêter puis garer et se garer. D'une façon générale, chaque unité polysémique est décrite sous la forme d'un réseau de significations ayant à sa racine un invariant de signification (ou «signifié de puissance» au sens de G. Guillaume (1969) et 
Picoche (1986) et aux sommets des significations liées entre elles par des spécifications de l'invariant sémantique ${ }^{\mathrm{ii}}$.

La polysémie d'un lexème verbal (ou d'une préposition) n'est pas, selon nous, adéquatement analysée par le seul jeu classificatoire de traits pertinents (sèmes) car, en se plaçant dans le cadre de la sémantique cognitive, la signification d'un lexème verbal (ou prépositionnel) est décrite sous la forme d'un schème composé de diverses relations emboîtées. Le schème associé à une unité verbale est une représentation structurée qui se présente comme un intermédiaire (comparable à la notion de schème chez Kant (1787 [2012]) entre d'un côté, un prédicat verbal (ou concept chez Kant), exprimé en français par un verbe et ses diverses places actancielles entretenant des relations casuelles avec lui, et d'un autre côté, de représentations sémantico-cognitives (représentations " sensibles » chez Kant) organisées par la perception de l'environnement et des actions, plus ou moins intentionnelles, portant sur l'environnement. La structuration interne de chaque schème fait appel à des primitives sémantico-cognitives qui sont étroitement liées à des catégorisations cognitives plus générales (ou types sémantiques) d'entités et à des schémas élémentaires de perception et d'action. Ces primitives sont candidats à être des « invariants universels $\gg{ }^{i i i}$, en nombre réduit, que l'activité de langage met en œuvre en les composant avec des primitives technico-culturelles plus spécifiques à chaque langue, afin d'exprimer publiquement les significations mentales qui sont sous-jacentes aux énoncés échangés entre les locuteurs d'une langue.

Notre analyse de la polysémie verbale s'inscrit dans une démarche où sémantique cognitive et sémantique formelle ne doivent pas être opposées car elles se complètent. Plus précisément, il s'agit du cadre théorique de la GRammaire Applicative, Cognitive des opérations Énonciative (GRACE) (Desclés 2011b, Desclés et al. 2016) qui présente une architecture cognitive et computationnelle où plusieurs niveaux de représentations sont reliées entre elles par des calculs explicites, ce qui permet d'associer des représentations sémantiques aux configurations morphosyntaxiques d'une langue. Dans ces analyses, le modèle de la GRACE fait appel à des organisations applicatives composées d'opérateurs appliqués à des opérandes (de différents types fonctionnels) et d'opérateurs abstraits, appelés combinateurs, de la Logique Combinatoire de Curry (Curry et al. 1958, 1972); ces combinateurs composent des opérateurs élémentaires afin de construire des schèmes et des représentations de significations. Dans ce modèle, les schèmes sémantico-cognitifs qui représentent, par des $\lambda$-expressions ${ }^{\text {iv }}$, des significations d'unités lexicales constitutives d'un dictum (c'est-à-dire une relation prédicative structurée, appelée aussi 'lexis') sont composés avec des opérations de "prise en charge énonciatives » relevant de la catégorie complexe du TAM, constitutives d'un modus, lequel est analysé comme un opérateur complexe qui, en s'appliquant au dictum, construit un énoncé, conformément à la décomposition de l'énoncé par Bally (1932/1965). Ce modèle vise à appréhender, dans une démarche unifiée, plusieurs courants :

(i) représentations sémantiques et cognitives qui font appel, souvent implicitement, à des conceptualisations topologiques, par exemple, au moyen des schèmes analytiques de Pottier $(2000,2012)$ ou des représentations iconiques de Langacker $(1987,1991)$ et de Talmy (1988);

(ii) analyse et représentation des opérations énonciatives dans le cadre de la théorie de l'énonciation de Bally (1932/1965), Benveniste (1966, 1974), Culioli (1990, 1999, 2002);

(iii) décompositions paraphrastiques dans le cadre de la Grammaire des opérateurs de Harris (1976) ;

(iv) analyses syntaxiques et sémantiques formalisées, à la suite de Shaumyan (1987), au moyen des combinateurs de la Logique Combinatoire de Curry 
(1958, 1972) et des Grammaires Catégorielles étendues (Desclés et Biskri, 1997).

A partir de problèmes précis, comme la polysémie, l'aspectualité et la temporalité grammaticalisées par les langues, et une analyse détaillée d'exemples illustratifs, nous cherchons à évaluer et à complexifier, quand c'est nécessaire, la pertinence du modèle. Nous renvoyons aux publications qui exposent ce modèle et ses développements, entre autres à Desclés et al. (2016). Le présent article n'exposera pas les développements techniques (par exemple, les calculs des significations) que les analyses proposées supposent lorsque l'on veut relier explicitement les énoncés à leurs représentations sémantiques. Il s'agit de montrer, en quelques pages, comment se construisent progressivement les schèmes et représentations interprétatives dans un traitement de la polysémie verbale.

\section{Primitives sémantico-cognitives}

Les principaux types d'entités catégorématiques sont des formes catégorielles étroitement liées à la perception, ce sont les types (ou catégories de base) : objets individuels, classes distributives d'objets individuels, classes collectives ou méréologiques et massives, informations et lieux. A chaque type, assigné à une entité linguistique, est associé un ensemble de propriétés. Ainsi, au type des objets individuels est associé la relation d'appartenance d'un objet à une classe distributive qui elle-même peut faire partie d'une autre classe distributive (Desclés 1987); au type des lieux sont associés des opérateurs topologiques comme prendre l'intérieur ('Int'), l'extérieur ('Ext'), la fermeture ('Fer') ou la frontière ('Fro') d'un lieu 'Loc'. Une même unité lexicale peut, selon ses contextes d'emploi, être appréhendée par différents types de base. Par exemple, l'unité lexicale 'bureau' est un objet individuel (transporter un bureau) ou un lieu spatial (dans un bureau) ou un lieu temporel (pendant le bureau) ou une classe collective (le bureau de l'assemblée) ou encore une activité (au bureau, on s'ennuie...). A côté des types de base des entités, l'étude sémantique utilise des primitives relationnelles de différents types fonctionnels (techniquement, ce sont les types fonctionnels de Church 1941) . $^{\mathrm{v}}$. Ces primitives relationnelles sont les éléments constitutifs des situations. Les différents relateurs de repérage (notés à l'aide du repérage générique Rep) entre entités de divers types sont constitutifs des situations statiques. Nous avons, par exemple, les situations statiques: d'appartenance $[\mathrm{x} \in \mathrm{C}$ ] ( $\mathrm{x}$ appartient à la classe distributive $\mathrm{C} »)$, de localisation $[\mathrm{x} \in$ Int (Loc) ] (« x appartient à l'intérieur du lieu Loc ») et [ $\mathrm{x} \in$ Fro (Loc) ] (« x appartient à la frontière du lieu Loc »). Les relateurs de mouvement (noté 'MOUVT') et de changement (noté 'CHANGT') sont constitutifs de situations cinématiques dans lesquelles une entité est affectée par un mouvement (par exemple, La mer monte) ou par un changement d'états (par exemple, La plage diminue). Les situations dynamiques sont construites à l'aide de l'effectuation et du contrôle d'un agent. Le relateur d'effectuation, noté 'FAIRE', établit une relation entre une entité qui effectue une action et la situation cinématique qui en résulte (par exemple, La mer détruit la plage). Le relateur de contrôle, noté 'CONTR', porte sur l'effectuation d'une situation cinématique ; le contrôle est exercé par une entité qui a acquis (ou possède généralement d'un point de vue ontologique) la capacité de « déclencher ou d'interrompre un mouvement ou un changement»; c'est cette propriété de contrôle qui caractérise essentiellement le rôle grammatical d'un agent ${ }^{\mathrm{vi}}$. L'agent peut contrôler l'action d'une entité instrumentale qui effectue une action en affectant un patient (Cette entreprise de travaux publics construit des digues avec une grue) ou contrôler ses propres actions sans aucun intermédiaire exprimé (Luc construit lui-même une digue pour sauver sa maison). A ces deux primitives constitutives de situations dynamiques, il convient d'ajouter la primitive de visée intentionnelle d'un but, notée 'TELEO'; cette 
dernière établit une relation directe entre un agent et la situation visée (Le maire souhaite protéger la plage par une digue). Si certaines entités sont naturellement agentives (ainsi, les humains sont ontologiquement agentifs), elles peuvent cependant perdre leur capacité de contrôle (Luc est tombé dans la fosse) et, inversement, une entité qui est ontologiquement non agentive peut être présentée dans le discours comme un agent (Pour se venger des hommes qui fouillent durant toute l'année dans ses entrailles, la mer - entité agentive - a pris la forme d'une tempête afin de pouvoir avaler les pêcheurs qui $s$ 'aventuraient sur ses eaux déchaînées). Les rôles grammaticaux d'agent et d'instrument ne doivent pas être assimilés à la notion de causalité puisque cette dernière s'établit entre deux situations et non entre une entité et une situation (Desclés et Guentchéva 1998); par exemple, Les mesures prises par le gouvernement diminuent le pouvoir d'achat des retraités (relation de causalité) doit être distingué de Le gouvernement oublie les retraités (relation agentive) $^{\mathrm{vii}}$.

Les primitives précédentes sont clairement liées aux capacités cognitives des humains qui ont appris, souvent par expérience, à distinguer différentes catégories de base (ou sortes) des entités avec toutes les propriétés qui leur sont attachées : objets individuels (un couteau, une chaise...), classes distributives (un ensemble de couteaux, de chaises), classes collectives (une armée, la police), objets massifs (de l'or, de l'eau...), lieux (une maison, un village...). Quant aux primitives 'MOUVT' et 'CHANGT', elles sont directement liées à la perception. L'homme a la capacité (sans doute innée) de percevoir et de catégoriser les différentes situations statiques ainsi que les différentes phases d'un mouvement ou d'un changement; par exemple, on perçoit et verbalise facilement les différents stades de l'évolution d'un enfant : il se met à grandir, il commence à grandir, il grandit, il finit de grandir, il termine de grandir, il a bien grandi, il est maintenant grand. Les primitives dynamiques 'FAIRE', 'CONTR' et 'TELEO' sont également liées aux capacités cognitives qui savent (ou non) déterminer l'origine d'une action, jugée contrôlée ou non, avec éventuellement un but spécifiquement visé.

\section{Schèmes temporalisés}

Les schèmes sont construits à l'aide des relateurs cinématiques et dynamiques portant sur différents types d'entités mises en relation. Ils sont présentés sous forme d'expressions applicatives préfixées où chaque opérateur s'applique à un opérande et il est positionné devant cet opérande (Desclés 1990 ; Desclés et al. 2016). Par exemple, la relation statique [ $\mathrm{x} \in \operatorname{Int}(\mathrm{Loc})]$ ( « $\mathrm{x}$ appartient à l'intérieur d'un lieu Loc ») est présentée sous la forme applicative préfixée : ' $\in$ (Int (Loc)) x'. Le changement de position spatiale d'un objet mobile comme 'sortir du lieu d'un objet accessible à la vision' (par exemple, dans L'avion disparaît de ma vue), est représentée par l'expression applicative :

'sortir du lieu d'un objet y accessible à la vision de $\mathrm{x}$ '

$$
\left.={ }_{\operatorname{def}} \operatorname{MOUVT}\left(\operatorname{SIT}^{1}[\mathrm{y}, \mathrm{x}]\right)\left(\operatorname{SIT}^{2}[\mathrm{y}, \mathrm{x}]\right)\right)
$$

avec : $\operatorname{SIT}^{1}[\mathrm{y}, \mathrm{x}]={ }_{\text {def }} \in \operatorname{Int}(\operatorname{Loc}($ lieu des objets accessibles à la vision de $\mathrm{x})) \mathrm{y}$ $\operatorname{SIT}^{2}[\mathrm{y}, \mathrm{x}]=_{\text {def }} \in \operatorname{Ext}($ Loc (lieu des objets accessibles à la vison de $\mathrm{x})$ ) $\mathrm{y}$

Ce schème n'est cependant pas complètement adéquat à la description de la signification de 'sortir du lieu d'un objet y accessible à la vision de x'. En effet, il est nécessaire de tenir également compte de la temporalité sous-jacente puisque tout mouvement " consomme du temps ». Aussi introduisons-nous la primitive aspectuelle sous la forme d'un opérateur aspectuel (désigné génériquement par ' $\mathrm{ASP}_{\mathrm{I}}$ ') ; en s'appliquant à une relation prédicative quelconque, désignée par ' $\Lambda$ ' (pour « lexis »), l'opérateur ' $\mathrm{ASP}_{\mathrm{I}}$ ' construit l'expression 'ASP $\mathrm{A}_{\mathrm{I}}(\Lambda)$ ' qui dénote une situation actualisée sur l'intervalle 
topologique 'I', supposé être continu car composé d'instants contigus et successifs. Dans le cas d'un état, l'intervalle d'actualisation est ouvert (noté 'O'), c'est-à-dire sans un premier, ni un dernier instant d'actualisation (ces instants sont exclus de l'intervalle ' $O$ ' d'actualisation); dans le cas d'un événement, l'intervalle d'actualisation est fermé (noté ' $F$ '), avec nécessairement un premier et un dernier instant d'actualisation (ces instants sont respectivement le début et la fin de l'événement) ; dans le cas d'un processus inaccompli, l'intervalle est semi-ouvert (noté ' $\mathrm{J}$ '), avec nécessairement un premier instant d'actualisation mais sans un dernier instant d'actualisation puisque le processus est saisi à un instant où il est inaccompli. Ainsi, une relation prédicative aspectualisée par l'un des opérateurs aspectuels ' $\mathrm{ETAT}_{0}$ ', ' $\mathrm{EVEN}_{\mathrm{F}}$ ' et 'PROC ${ }_{\mathrm{J}}$ ' (qui spécifient l'opérateur 'générique ' $\mathrm{ASP}_{\mathrm{I}}$ ') renvoie à une situation actualisée sur l'un des intervalles topologiques respectifs 'O', 'F' et 'J'.

Prenons quelques exemples simples d'aspectualisation. L'acte d'énonciation est toujours un processus inaccompli, que l'on désigne par l'opérateur composé ' $\mathrm{PROC}_{\mathrm{J} 0} \mathbf{0}$ (JE-DIS)' ou plus simplement par 'ENONC ${ }_{\mathrm{J} 0}$ '. Lorsque cet opérateur d'énonciation s'applique à une relation prédicative aspectualisée ' $\operatorname{ASP}_{\mathrm{I}}(\Lambda)$ ', il traduit une «prise en charge » de ' $\operatorname{ASP}_{\mathrm{I}}(\Lambda)$ ' par l'énonciateur 'JE' actualisée sur l'intervalle (qualitatif) d'énonciation ' $\mathrm{J}_{0}$ ', dont la borne droite ' $\mathrm{d}\left(\mathrm{J}_{0}\right)$ ' est souvent désignée par ' $\mathrm{T}^{\circ}$ '. Remarquons que la borne ' $\mathrm{T}$ ' $\mathrm{n}$ 'est évidemment pas « l'instant d'énonciation »; elle est simplement la borne d'inaccomplissement de l'énonciation (en cours) dans le référentiel énonciatif ${ }^{\text {viii }}$. Un événement s'inscrit le plus souvent dans une séquence événementielle ( $J$ 'ai déjeuné, puis je suis allé me promener). L'état résultant (J'ai déjeuné. Je n'ai plus faim) ${ }^{\mathrm{ix}}$, présenté comme concomitant à son énonciation, est actualisé sur un intervalle ouvert qui est postérieur à l'intervalle fermé d'actualisation de l'événement qui a donné naissance à cet état résultant. Il existe, à côté de l'état résultant, d'autres états. Prenons, par exemple, L'avion est en vol; il s'agit d'un état d'activité (de l'avion) qui s'oppose à d'autres activités et à d'autres états : 'être en réparation', 'être arrivé', 'être au parking'... ; cet état d'activité indique en même temps l'existence d'un processus sous-jacent ( $L$ 'avion est en train de voler ou $L$ 'avion vole) qui est actualisé sur l'intervalle semi-ouvert 'J' (Desclés et Guentchéva 1995); l'intervalle d'actualisation ' $\mathrm{O}$ ' de l'état d'activité 'est en vol' est nécessairement contenu dans l'intervalle 'J' puisque, par définition, l'état d'activité exclut les phases du commencement et de la fin du processus et, en outre, il néglige toute variation interne, ce qui est confirmé par le test linguistique suivant: alors que l'on a L'avion commence à voler / L'avion vole de mieux en mieux (ou de plus en plus vite), on n'a pas *L'avion commence à être en vol / * L'avion est en vol de mieux en mieux (ou de plus en plus vite).

Revenons au schème cinématique 'sortir du lieu d'un objet y accessible à la vision de $\mathrm{x}$ '. Une représentation plus adéquate doit tenir compte des conditions temporelles portant sur les intervalles $\mathrm{O}_{1}$, $\mathrm{F}$ et $\mathrm{O}_{2}$ :

'sortir du lieu d'un objet y accessible à la vision de x'

$$
={ }_{\text {def }} \operatorname{EVEN}_{\mathrm{F}}\left(\operatorname{MOUVT}_{\mathrm{F}}\left(\operatorname{SIT}_{\mathrm{O} 1}[\mathrm{y}, \mathrm{x}]\right)\left(\mathrm{SIT}_{\mathrm{O} 2}[\mathrm{y}, \mathrm{x}]\right)\right)
$$

$$
\begin{aligned}
& \text { avec: } \left.\left.\operatorname{SIT}^{1} \mathrm{O} 1 \mathrm{y}, \mathrm{x}\right]\right)={ }_{\operatorname{def}} \operatorname{ETAT}_{\mathrm{O} 1}\left(\operatorname{SIT}^{1}[\mathrm{y}, \mathrm{x}]\right) \text {; } \\
& \left.\operatorname{SIT}^{2}{ }_{2}[\mathrm{y}, \mathrm{x}]\right)=_{\text {def }} \operatorname{ETAT}_{\mathrm{O} 2}\left(\operatorname{SIT}^{2}[\mathrm{y}, \mathrm{x}]\right) \\
& {\left[\mathrm{g}(\mathrm{F})=\mathrm{d}\left(\mathrm{O}_{1}\right)\right] \&\left[\mathrm{~g}\left(\mathrm{O}_{2}\right)=\mathrm{d}(\mathrm{F})\right]}
\end{aligned}
$$

Les deux relations temporelles précisent que : $1^{\circ}$ ) la borne gauche ' $\mathrm{g}(\mathrm{F})$ ' de ' $\mathrm{F}$ ' est identifiée à la borne droite ' $\mathrm{d}\left(\mathrm{O}_{1}\right)^{\prime}$ ' de $\mathrm{O}_{1}$ qui précède immédiatement ' $\mathrm{F}$ '; $2^{\circ}$ ) la borne gauche ' $\mathrm{g}\left(\mathrm{O}_{2}\right)$ ' de $\mathrm{O}_{2}$, qui suit immédiatement $\mathrm{F}$, est identifiée à la borne droite ' $\mathrm{d}(\mathrm{F})$ ' de $\mathrm{F}$. La temporalité sous-jacente au schème est supposée continue (c'est-à-dire sans aucun saut entre deux instants consécutifs et sans aucune lacune entre deux instants consécutifs); ces bornes sont des « coupures continues » (au sens de Dedekind ${ }^{\mathrm{x}}$ ) dans l'union des intervalles 
$\mathrm{O}_{1} \cup \mathrm{F} \cup \mathrm{O}_{2}$ : la borne gauche $\mathrm{g}(\mathrm{F})$ et la borne droite $\mathrm{d}(\mathrm{F})$ sont des bornes fermées dans ' $\mathrm{F}$ ' mais ces bornes, identifiées à respectivement $\mathrm{d}\left(\mathrm{O}_{1}\right)$ et $\mathrm{g}\left(\mathrm{O}_{2}\right)$, sont nécessairement ouvertes dans les intervalles $\mathrm{O}_{1}$ et $\mathrm{O}_{2}$ puisque la suite des instants est supposée continue.

La notion de mouvement est une forme de changement. Le changement peut s'opérer entre un état initial d'activité d'un objet (ce qui exclut le début et la fin du processus sousjacent à l'état d'activité) et une absence d'activité du même objet. Ce changement peut être exprimé sous la forme d'une $\lambda$-expression applicative (où l'opérateur ' $\lambda y$ ' lie toutes les occurrences de la variable ' $y$ ' dans l'expression) :

'annulation du mouvement d'un objet'

$$
={ }_{\text {def }} \lambda \mathrm{y} \cdot\left\{\operatorname{EVEN}_{\mathrm{F}}\left(\mathrm{CHANGT}_{\mathrm{F}}\left(\operatorname{SIT}^{1}{ }_{\mathrm{O} 1}[\mathrm{y}]\right)\left(\operatorname{SIT}_{\mathrm{O} 2}^{1}[\mathrm{y}]\right)\right)\right\}
$$

avec

$$
\begin{aligned}
& \operatorname{SIT}^{1} \mathrm{O}[\mathrm{y}]=\text { def } \operatorname{ETAT}_{\mathrm{O} 1}(\text { est en mouvement }(\mathrm{y})) \\
& \left.\mathrm{SIT}_{\mathrm{O} 2}^{2}[\mathrm{y}]\right)={ }_{\text {def }} \operatorname{ETAT}_{\mathrm{O} 2}(\mathrm{NON}(\text { est en mouvement }(\mathrm{y}))) \\
& {\left[\mathrm{g}(\mathrm{F})=\mathrm{d}\left(\mathrm{O}_{1}\right)\right] \&\left[\mathrm{~g}\left(\mathrm{O}_{2}\right)=\mathrm{d}(\mathrm{F})\right]}
\end{aligned}
$$

Cette $\lambda$-expression est un schème qui peut être représenté, par ailleurs et de façon entièrement équivalente, sous la forme de relations emboîtées, ou bien à l'aide d'un diagramme bi-dimensionnel dans lequel l'axe horizontal représente la suite continue des instants et l'axe vertical les différents états d'un même objet (voir la Figure 1).

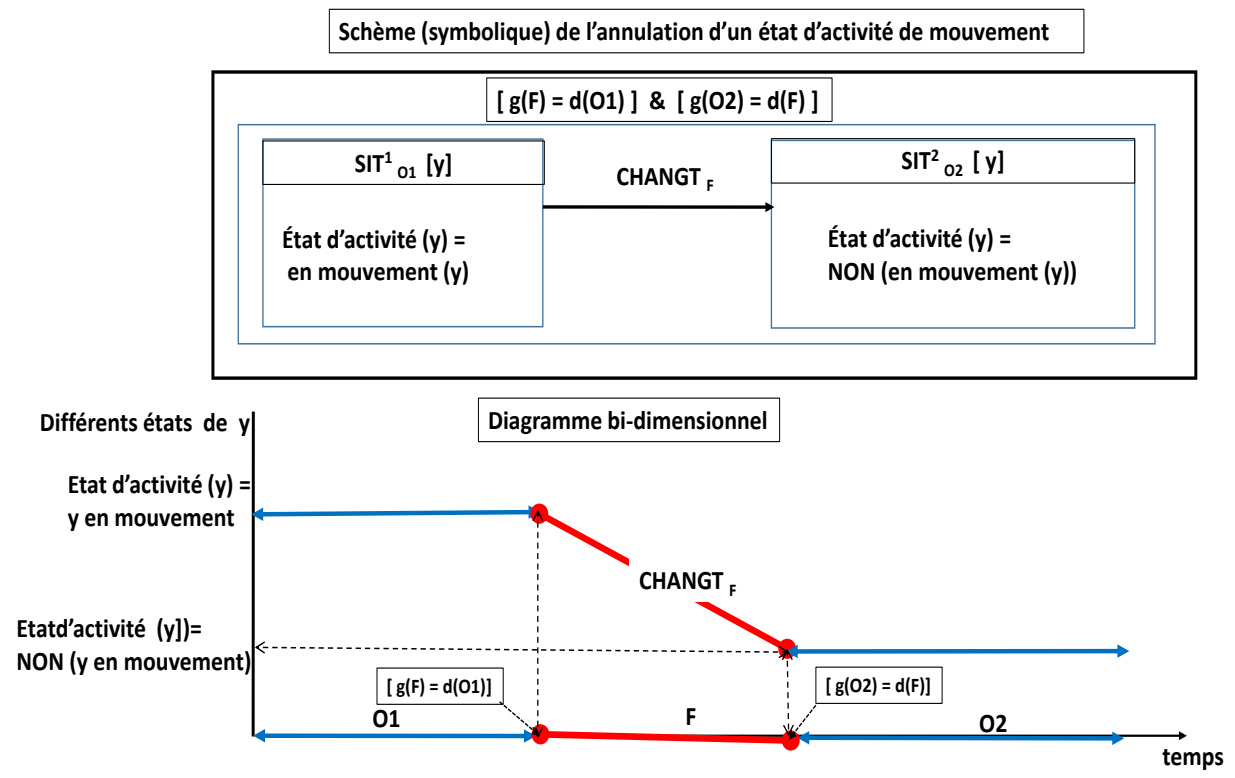

Figure 1 : Schème symbolique et diagramme bi-dimensionnel d'un changement d'états d'un objet

\section{Les schèmes de 'arrêter' et 's'arrêter'}

Le schème qui représente la signification du lexème verbal 'arrêter' dans :

\section{(1) Le feu rouge arrête la voiture.}

est exprimé par la $\lambda$-expression : 


\section{$\lambda z . \lambda y .\left\{\operatorname{EVEN}_{\mathrm{F}}\left(\mathrm{FAIRE}_{\mathrm{F}}\left(\mathrm{EVEN}_{\mathrm{F}}\right.\right.\right.$ ('annulation du mouvement d'un objet' (y))) z) $\}$}

En composant les relateurs (par l'opérateur de composition fonctionnelle ' 0 '), on obtient finalement le schème dynamique transitif événementiel de 'arrêter le mouvement d'un objet' (correspondant à l'énoncé (1)) :

$$
\lambda z . \lambda y .\left\{\left(\operatorname{EVEN}_{\mathrm{F}} \text { o } \mathrm{FAIRE}_{\mathrm{F}}\right)\left(\left(\mathrm{EVEN}_{\mathrm{F}} \mathbf{0} \mathrm{CHANGT}_{\mathrm{F}}\right)\left(\operatorname{SIT}_{01}^{1}[\mathrm{y}]\right)\left(\operatorname{SIT}_{02}^{1}[\mathrm{y}]\right)\right) \mathrm{z}\right\}
$$

L'interprétation du schème (I) est: «un objet (non agentif) ' $z$ ' est à l'origine du changement actualisé sur l'intervalle temporel ' $F$ ' ; ' $z$ ' fait passer l'objet ' $y$ ' de l'état de mouvement à une absence de mouvement ». Lorsque dans ce schème, les variables sont instanciées par respectivement 'le-feu-rouge' et 'la-voiture', on obtient la représentation sémantico-cognitive de (1) :

$\left(\mathrm{EVEN}_{\mathrm{F}} \mathbf{0} \mathrm{FAIRE}_{\mathrm{F}}\right)$

$\left(\left(\mathrm{EVEN}_{\mathrm{F}} \mathbf{0} \mathrm{CHANGT}_{\mathrm{F}}\right)\left(\mathrm{SIT}^{1}{ }_{01}\right.\right.$ [la-voiture $\left.]\right)\left(\mathrm{SIT}_{02}^{1}\right.$ [la-voiture $\left.\left.]\right)\right)($ le-feu-rouge $)$

Prenons maintenant l'exemple :

(2) La police arrête une voiture.

Dans cet énoncé, l'introduction d'un agent (présenté comme une classe collective) conduit à complexifier le schème précédent puisque l'agent (la police) contrôle l'effectuation du changement qui affecte le mouvement d'un patient (un objet), d'où le schème dynamique agentif transitif événementiel :

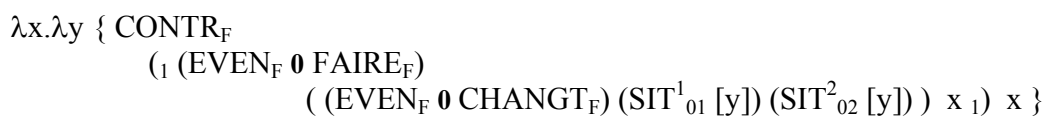$$
\left.\left(\left(\operatorname{EVEN}_{\mathrm{F}} \mathbf{0 ~ C H A N G T}_{\mathrm{F}}\right)\left(\mathrm{SIT}_{01}^{1}[\mathrm{y}]\right)\left(\mathrm{SIT}_{02}^{2}[\mathrm{y}]\right)\right) \mathrm{x}_{1}\right) \mathrm{x} \text { \} }
$$

Ce schème indique que l'agent ' $x$ ' contrôle sa propre effectuation de l'action 'arrêter une entité y’. Il peut être présenté par des relations emboittées (Figure 2) :

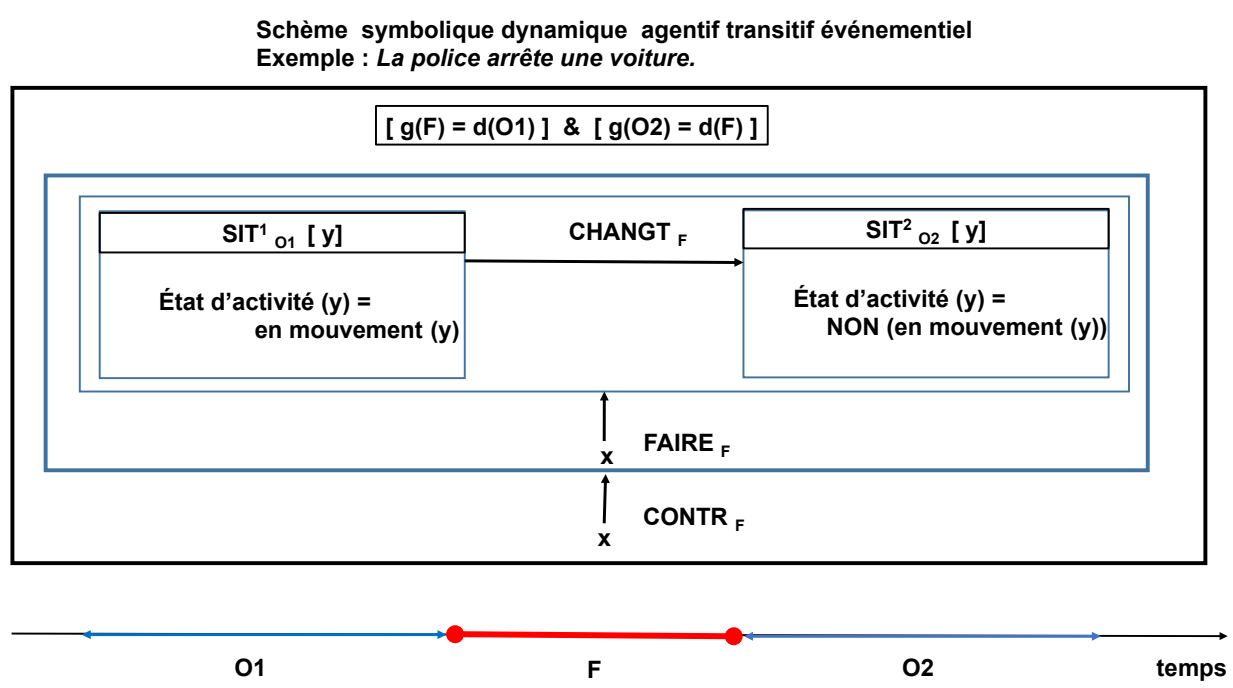

Figure 2: Schème transitif où un agent ' $x$ ' contrôle le changement affectant un patient ' $y$ ' 
L'instanciation des variables du schème précédent par respectivement 'la-police' et 'une voiture', représente la signification de la phrase (2) :

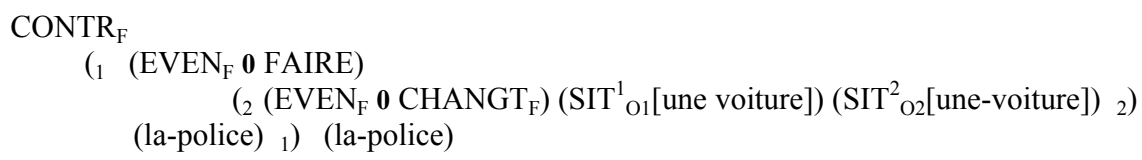

Cette représentation symbolique applicative peut être comparée à la représentation que l'on obtiendrait dans le cadre des représentations figuratives de Pottier $(2000,2012)$ qui s'inspire directement des schèmes de « la théorie des catastrophes » de Thom $(1981,1983)$ (Figure 3) :
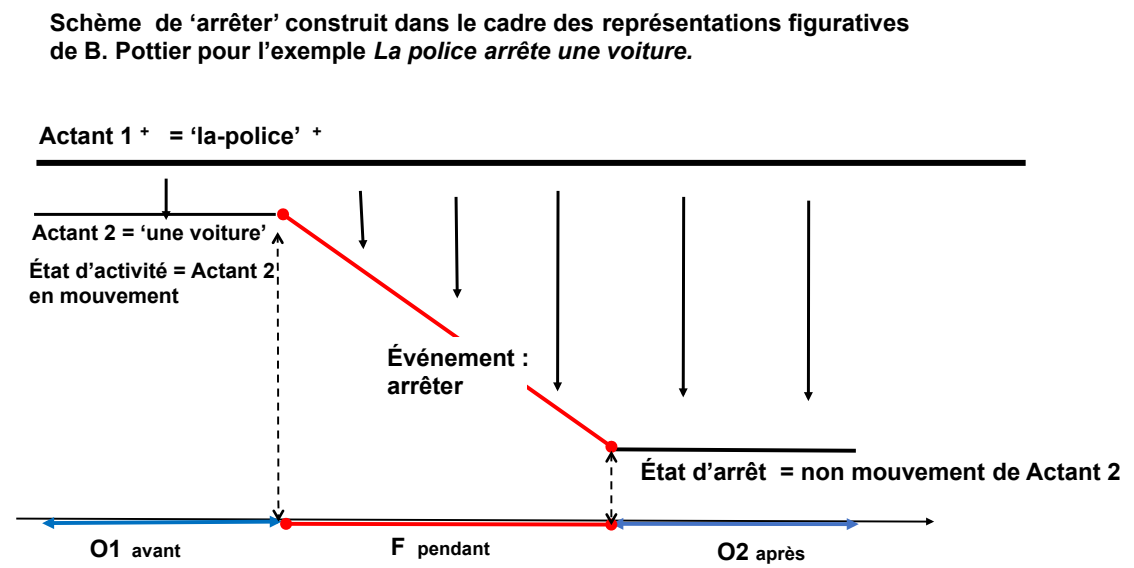

Figure 3 : Schème figuratif d'une prédication transitive

Lorsque l'agent effectue son action d'arrêter, il peut faire appel à un instrument intermédiaire, d'où le schème dynamique agentif transitif événementiel avec instrument :

\section{$\lambda x . \lambda y . \lambda u\left\{\right.$ CONTR $_{\mathrm{F}}$}

$$
\begin{aligned}
& \text { (1 } \quad\left(\mathrm{EVEN}_{\mathrm{F}} \mathbf{0}\right. \text { FAIRE) } \\
& \text { ( } 2\left(\mathrm{EVEN}_{\mathrm{F}} \mathbf{0} \text { CHANGT) }\left(\mathrm{SIT}^{1}{ }_{01}[\mathrm{y}]\right)\left(\mathrm{SIT}^{2}{ }_{02}[\mathrm{y}]\right)_{2}\right) \\
& \left.\left.\mathrm{u}_{1}\right) \mathrm{x}\right\}
\end{aligned}
$$

Ce schème signifie : « un agent ' $x$ ' contrôle l'effectuation, au moyen d'un instrument ' $u$ ', du changement qui affecte un patient ' $y$ ' qui passe d'un état d'activité de mouvement à un état de non mouvement. ». Ce schème, auquel on peut associer des relations emboîtées, représente la signification du verbe arrêter (avec trois actants) dans la phrase :

(3) La police arrête les voitures avec un barrage.

Prenons maintenant un autre exemple : 
(4) Luc s'arrête.

Dans cet exemple, le schème du verbe s'arrête est :

$\lambda x .\left\{\right.$ CONTR $_{\mathrm{F}}$

(1 $\quad\left(\mathrm{EVEN}_{\mathrm{F}} \mathbf{0} \mathrm{FAIRE}_{\mathrm{F}}\right)$

$$
\begin{aligned}
& \left.{ }_{2}\left(\operatorname{EVEN}_{\mathrm{F}} \mathbf{0} \mathrm{CHANGT}_{\mathrm{F}}\right)\left(\operatorname{SIT}^{1}{ }_{\mathrm{O} 1}[\mathrm{x}]\right)\left(\mathrm{SIT}_{\mathrm{O} 2}^{2}[\mathrm{x}]\right){ }_{2}\right) \\
& \left.\mathrm{x}_{1}\right\}
\end{aligned}
$$

Ce schème signifie que l'agent ' $x$ ' effectue un contrôle sur l'action de changement qui l'affecte lui-même, d'où le changement entre les situations $\operatorname{SIT}^{1}{ }_{\mathrm{O} 1}[\mathrm{x}]$ et $\mathrm{SIT}^{2} \mathrm{O} 2[\mathrm{x}]$.

L'énoncé :

(5) Luc s'arrête dans la cour

apporte une spécification supplémentaire avec l'indication du lieu où s'arrête 'Luc'. Dans ce cas, le schème de $s$ 'arrête dans devient :

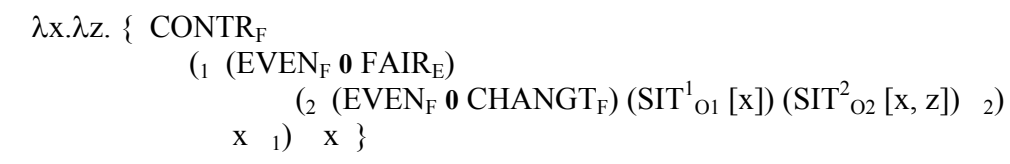

avec : $\operatorname{SIT}_{\mathrm{O} 2}^{2}[\mathrm{x}, \mathrm{z}]=_{\mathrm{def}} \quad\left[\operatorname{SIT}_{\mathrm{O} 2}^{2}[\mathrm{x}] \&[\mathrm{x} \in \operatorname{Int}(\operatorname{Loc}(\mathrm{z})]]\right.$

Le schème $(\mathrm{V})$ signifie que l'agent ' $\mathrm{x}$ ' qui est initialement en mouvement, contrôle le changement de son propre état d'activité vers une absence de mouvement qui est finalement localisée à l'intérieur d'un lieu 'Loc(z)'. Ainsi, dans l'exemple (5), le lieu exprimé par dans la cour est une instanciation de l'intérieur 'Int (Loc(z))'.

\section{Réseau polysémique de 'arrêter' et ' $s$ 'arrêter'}

Les exemples précédents (1) - (5) sont relatifs à l'arrêt d'un état d'activité du mouvement spatio-temporel d'un objet. Pour les mêmes lexèmes verbaux 'arrêter' et 's'arrêter', les schèmes spatiaux peuvent être étendus à des changements d'états d'activité qui ne sont plus des mouvements spatiaux, mais diverses activités qui concernent un objet, comme dans les exemples suivants :

(6) La tempête arrête le match.

(7) La police arrête une bande de voleurs.

(8) Le juge arrête les vols dans la cité par des jugements sévères.

(9) Luc s'arrête de travailler.

(10) Arrivé au bureau, Luc s'arrête de plaisanter.

Dans ces exemples, les verbes arrête et $s$ 'arrête concernent des cessations d'activités non spatiales : un match en cours suspendu par la tempête (6); une activité de vol attribuée à une classe collective (7); les activités de vols continuels dans la cité par l'intermédiaire de jugements sévères (8); l'activité de travail de 'Luc' (9); l'interruption d'une activité usuelle de 'Luc' dès qu'il se trouve au bureau (sans doute pour mieux travailler). Les schèmes correspondants à ces emplois sont structurés d'une façon très analogue aux 
schèmes avec cessation de mouvement, la nature de l'activité étant spécifiée par le contexte, d'où les extensions des schèmes précédents $(\mathrm{I})$ - $(\mathrm{V})$ par les schèmes (I') - (V') que nous ne reproduisons pas ici. Ces différents schèmes ont cependant un invariant sémantique en commun : " annuler une activité concernant une entité», cette entité pouvant être un objet individuel (comme 'un voleur') ou une situation événementielle unique (comme 'le match') ou plurielle (comme 'des vols dans la cité'). Nous en déduisons le réseau polysémique des lexèmes arrêter et s'arrêter sous la forme d'un réseau de significations représentées par des schèmes et illustrés par des exemples (Figure 4). Tous les schèmes du réseau polysémique héritent de l'invariant sémantique; les flèches entre les schèmes sont des relations de spécification qui viennent préciser certaines conditions par rapport au schème supérieur, par exemple, des identifications de variables (un agent peut être en même temps un instrument) ou des conditions sur les types des entités argumentales des relations constitutives d'un schème.

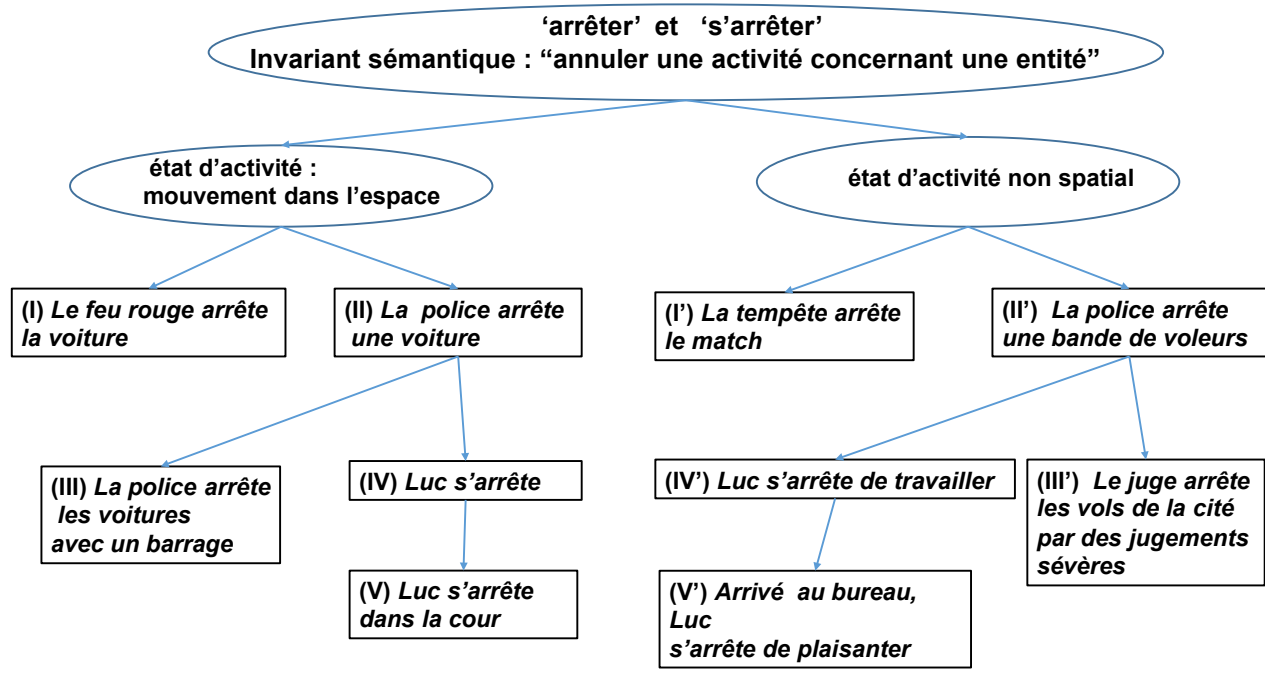

Figure 4 : Réseau polysémique (simplifié) de 'arrêter' et 's'arrêter'

Les autres emplois rencontrés dans la littérature exemplifient l'un des schèmes du réseau ou donnent une spécification plus particulière à l'un de ces schèmes. Prenons par exemple :

\section{(11) Le gouvernement arrête un décret.}

Dans (11), le gouvernement (qui est une entité agentive collective) décide d'arrêter les éventuels changements de formulations de propositions juridiques pour adopter une proposition qui, devenue décret, devient stable et n'est plus modifiable. Cet énoncé est une exemplification plus spécifique du schème (II') de la figure 4. Prenons maintenant :

\section{(12) Le regard de Luc s'est arrêté sur les mains de la Joconde.}

Cet énoncé signifie que 'le regard' se déplace dans l'espace pour venir s'immobiliser à la frontière d'un lieu très précis qui est bi-dimensionnel ('sur les mains de la Joconde') ; cet énoncé relève alors du schème $(\mathrm{V})$. Il est possible de reprendre toutes les classifications 
proposées par Lewicka, Bogacki et al. (1983), avec nos propres primitives du cadre théorique qui est exposé ici.

\section{Schèmes de 'garer' et 'se garer'}

La consultation des dictionnaires (par exemple, Le Robert historique 1992: 871) nous apprend que le verbe garer était un terme du vocabulaire maritime qui signifiait « amarrer un navire », puis «mettre à l'abri un bateau». Quant au lexème gare, il signifiait antérieurement «distance» et "désignait la partie d'une rivière ou d'un canal où les bateaux peuvent se croiser, se garer ». Ces termes ont évolué avec les technologies des transports (le train entre en gare / sort de la gare). Le terme de garage est ensuite entré dans le champ sémantique des véhicules. Ainsi, sortir sa voiture d'un garage tend à signifier que l'on sort sa voiture d'un lieu où elle était (éventuellement) protégée, pour la mettre, par exemple, en activité. En synchronie, gare, garage et (se) garer sont ressentis comme étant sémantiquement reliés. Prenons l'exemple suivant :

\section{(13) Luc gare sa voiture.}

L'énoncé (13) indique que l'agent 'Luc' contrôle et effectue une action qui change l'état d'activité du mouvement de l'objet mobile 'la voiture', pour aboutir à une absence complète de mouvement, en ajoutant une visée intentionnelle de l'agent : le changement spatial doit positionner l'objet dans un lieu où des objets analogues peuvent y être rangés. Le schème qui représente la signification du verbe 'gare' dans (13), exprime ainsi donc deux informations coordonnées : d'une part, un changement qui conduit à une absence de mouvement et, d'autre part, la visée par l'agent d'un but. Cette visée téléonomique est exprimée par le relateur TELEO dans la relation :

$$
\operatorname{TELEO}_{\mathrm{F}}\left(\operatorname{ETAT}_{\mathrm{O} 2}(\operatorname{Rep}(\operatorname{Loc}(\mathrm{z})) \mathrm{y}) \mathrm{x}\right.
$$

Cela signifie que «l'agent ' $x$ ' vise que l'objet ' $y$ ' soit repéré par rapport à un lieu déterminé 'Loc $(\mathrm{z})$ ' ». Le schème (présenté avec une notation applicative préfixée avec l'opérateur de conjonction ' $\&$ ') est le suivant :

$$
\begin{aligned}
& \lambda x . \lambda y . \lambda z\left\{\& \left({ } _ { 0 } \operatorname { C O N T R } _ { \mathrm { F } } \left({ }_{1}\left(\mathrm{EVEN}_{\mathrm{F}} \boldsymbol{0} \mathrm{FAIRE}_{\mathrm{F}}\right)\right.\right.\right. \\
& \left({ }_{2}\left(\mathrm{EVEN}_{\mathrm{F}} \mathbf{0} \mathrm{CHANGT}_{\mathrm{F}}\right)\left(\mathrm{SIT}_{01}^{1}[\mathrm{y}]\right)\left(\mathrm{SIT}_{02}^{2}[\mathrm{y}]\right){ }_{2}\right) \\
& \left.\begin{array}{llll}
x_{1} & x_{0}
\end{array}\right) \\
& \left(\operatorname{cELEO}_{\mathrm{F}}\left(\left[\left(\mathrm{SIT}_{02}^{2}[\mathrm{y}]\right) \&\left(\operatorname{ETAT}_{\mathrm{O} 2}(\operatorname{Rep}(\operatorname{Loc}(\mathrm{z})) \mathrm{y})\right]\right) \mathrm{x} \quad{ }_{0}\right)\right\}
\end{aligned}
$$

$$
\begin{aligned}
\text { avec : } & \operatorname{SIT}^{1}{ }_{\mathrm{O} 1}[\mathrm{y}]=\text { def } \operatorname{ETAT}_{01}(\text { est en mouvement }(\mathrm{y})) \\
& \left.\operatorname{SIT}_{\mathrm{O} 2}^{1}[\mathrm{y}]\right)=\text { def } \operatorname{ETAT}_{\mathrm{O} 2}(\mathrm{NON}(\text { est en mouvement }(\mathrm{y}))) \\
& {[\mathrm{g}(\mathrm{F})=\mathrm{d}(01)] \&[\mathrm{~g}(02)=\mathrm{d}(\mathrm{F})] }
\end{aligned}
$$

Ce schème est organisé par les relations emboîtées, la situation finale devenant un opérande commun au changement et à la visée téléonomique par un agent d'un but à atteindre (voir la figure 5). 
Schème symbolique dynamique agentif transitif événementiel de 'garer' :

Exemple : Luc gare sa voiture.

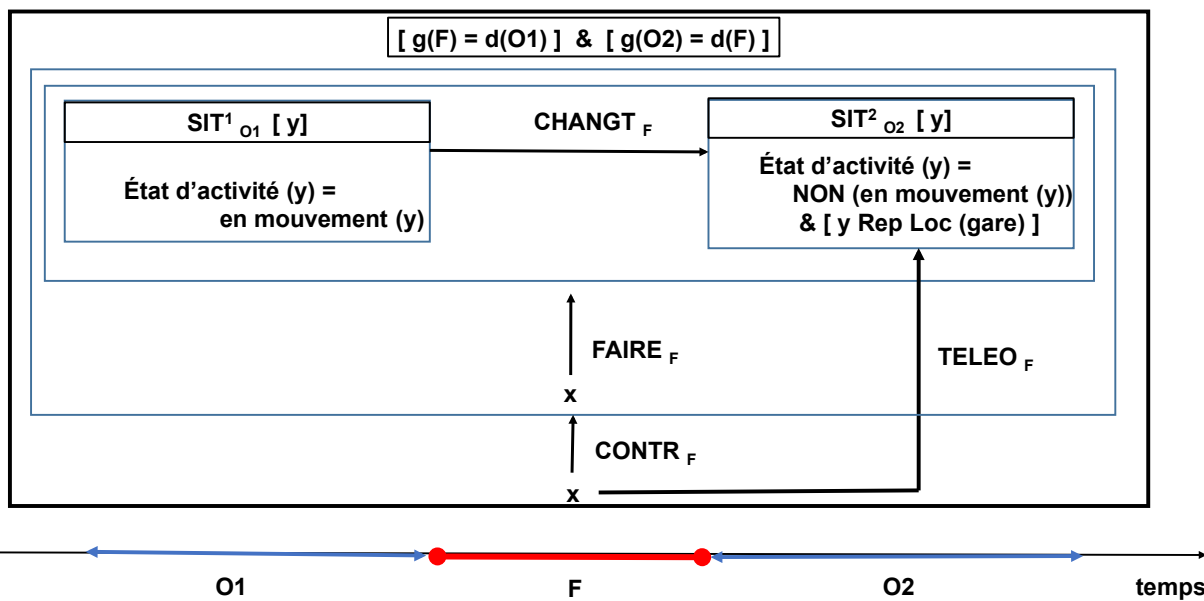

Figure 5 : Schème transitif avec la visée téléonomique d'une situation finale

Prenons le schème de 'se garer' dans un exemple comme :

(14) Luc se gare dans la rue.

Le schème se déduit du précédent puisque l'entité qui rejoint le lieu visé est l'agent luimême. Remarquons toutefois que l'agent est parfois implicite, comme dans :

(15) Le bus se gare près de l'arrivée des voyageurs.

où il est indiqué que l'objet 'le bus' se gare en un lieu précis en héritant des capacités d'un agent qui est, par ailleurs, facilement reconstructible ('le conducteur du bus') : 'le-bus', catégorisé ontologiquement comme un objet simplement mobile, devient, par l'activité discursive, un agent qui contrôle son propre mouvement dirigé volontairement vers une situation spatiale précise.

Comparons maintenant les énoncés suivants entre eux ${ }^{\mathrm{xi}}$ :

(16) Luc gare sa voiture sur un passage-piétons.

(17) Luc se gare sur un passage-piétons.

(18) Luc arrête sa voiture sur un passage-piétons.

(19) Luc s'arrête sur un passage-piétons.

Les énoncés (16) et (17) engagent beaucoup plus la responsabilité de l'agent que celle de l'agent dans les énoncés (18) et (19). Dans les deux premiers énoncés, la situation finale est présentée comme mieux installée avec une certaine stabilité temporelle; l'échelle d'acceptabilité (au sens de Harris) appliqué à (20) et (21) confirme cette différence sémantique :

(20) Luc s'arrête (/??? se gare) à un passage à niveau lorsque le feu passe au rouge.

(21) La voiture s'est arrêtée (/? s'est garée) au feu rouge. 
La signification de se garer fait en général appel à des lieux beaucoup mieux déterminés que celle qui est indiquée par l'emploi de s'arrêter :

(22) Luc s'arrête (/ ?? se gare) à Gap pour se reposer pendant son voyage vers le ski.

(23) Luc se gare (/s'arrête) dans le parking réservé aux clients de l'hôtel.

Comme arrêter et s'arrêter, les verbes garer et se garer ne sont pas réductibles aux seuls mouvements spatiaux ; ils sont également employés pour indiquer que l'on veut mettre des objets dans des lieux susceptibles de bénéficier d'une certaine protection (et d'éviter ainsi qu'ils soient parfois "égarés »), ou d'être positionnés en dehors d'activités jugées néfastes :

(24) Luc gare (*arrête) sa conférence déjà rédigée dans le tiroir de son bureau.

(25) Luc gare (*arrête) son argent dans un coffre.

(26) Il faut se garer (*s'arrêter) des mauvaises fréquentations.

(27) Ce spéculateur se gare (? arrête) maintenant des jeux de bourse qui l'ont pourtant enrichi.

(28) Diable, sorciers, fauves et brigands, garez vous, le fils d'Amadou Ali arrive.

(Bâ Amadou Hampâté, Amkoullel, l'enfant peul, 1991, p. 423)

(30) Garez vos miches cria quelqu'un, ça va saigner.

(Fallet, René, La grande ceinture, 1956, p. 76)

On peut multiplier les exemples en consultant les bases telles que Frantext ou les dictionnaires (Le Robert, Le Trésor de la langue française...). Dans ces emplois non spatiaux, garer et se garer se distinguent nettement de arrêter et de s'arrêter. L'invariant sémantique qui se dégage de leurs différents emplois renvoie à une notion de changement dont la situation finale est une visée téléonomique dans laquelle l'entité, qui a cessé toute activité, sera mieux protégée que si elle n'y était pas.

\section{Quelques remarques en guise de conclusion}

Nous avons cherché à opposer les verbes arrêter et s'arrêter aux verbes sémantiquement proches garer et se garer en montrant qu'ils ont en commun la notion de changement d'une activité (qui n'est pas nécessairement une activité de mouvement), orienté vers la cessation de cette activité. Lorsqu'ils s'opposent, en tant que synonymes, la nature de la visée téléonomique de l'agent (parfois implicite) de la situation finale les distingue assez nettement. Plutôt que de procéder à une étude fouillée de la polysémie et de la synonymie de ces verbes, nous avons préféré insister, dans cet article, sur la méthode de construction et de représentation des schèmes à l'aide de primitives relationnelles très générales et de types sémantico-cognitifs de base déterminés par des processus cognitifs (de perception et d'action plus ou moins intentionnelle) plus généraux. Ces primitives tendent à constituer des «invariants de l'activité de langage» qui transcenderaient la diversité des catégorisations opérées par une langue; chaque langue organise par des schèmes qui lui sont spécifiques, les significations lexicales et grammaticales. Les primitives relationnelles (de changement, d'effectuation, de contrôle et de téléonomie) permettent d'organiser et de représenter les rôles sémantiques des différentes entités actancielles dépendantes des prédicats verbaux. Ces rôles grammaticaux sont représentés par des relations qui établissent des liens entre des entités et des situations emboîtées dans une situation plus globale. Remarquons que les primitives relationnelles ne relient pas directement les entités actancielles entre elles comme le laisserait croire l'analyse directe des rôles sémantiques (souvent qualifiées de "relations casuelles») des différentes unités actancielles d'un prédicat verbal. Par exemple, le schème de la «transitivité sémantique » ne relie pas directement l'actant-agentif, exprimé par un sujet syntaxique, à l'actant-patient, exprimé 
par un objet direct syntaxique, puisque l'agent contrôle une situation dans laquelle le patient est impliqué. La structuration des schèmes, sous la forme de relations emboîtées, est plus complexe que la structuration des prédicats verbaux accompagnés de leurs actants ayant des rôles différents décrits souvent dans la littérature par un simple jeu d'étiquettes ('nominatif', 'accusatif', 'ergatif', 'instrument', 'locatif'...), sans toujours recevoir des définitions opératoires car l'étiquette ne fait pas le concept. C'est la structuration interne des schèmes qui permet de décrire, sous forme de relations, les rôles sémantiques des actants d'une relation prédicative. Les réseaux polysémiques tendent à montrer que la notion de « ressemblance » au sens de Wittgenstein (1922/1993), n'est pas assez opératoire pour mener une analyse fouillée de la polysémie. En effet, deux verbes comme se garer et s'arrêter, peuvent être sémantiquement proches et «se ressembler» seulement pour certaines valeurs des réseaux polysémiques respectifs mais rester assez éloignées pour les autres valeurs. Il s'agit donc de construire les réseaux polysémiques de chaque signifiant verbal, puis de les comparer, en faisant apparaître pour certaines valeurs de ces réseaux des schèmes communs qui permettraient d'expliquer la synonymie entre certains emplois contextualisés de ces verbes. Ainsi, deux verbes ne sont jamais globalement synonymes, ils ne le sont que pour certaines valeurs.

Les schèmes présentés dans cet article sont des schèmes événementiels qui sont actualisés sur des intervalles fermés d'instants. Les significations d'autres verbes, comme courir, marcher, grandir, vieillir, s'enrichir, se développer..., sont analysées par des schèmes processuels où aucun terme n'est a priori impliqué, à moins que le contexte n'ajoute certaines informations qui orientent vers un certain accomplissement (comme dans courir jusqu'à l'école, grandir jusqu'à avoir la taille d'un adulte, vieillir jusqu'à être devenu vieux...). D'autres expressions verbales, comme être naturellement courageux, être par essence honnête..., ont des significations décrites par des schèmes statiques actualisés sur des intervalles ouverts, par conséquent sans prise en compte d'une borne initiale et d'une borne finale fermées, lesquelles indiqueraient un premier et un dernier instant d'actualisation, ce qui ne veut évidemment pas dire que l'intervalle ouvert ne puisse pas être compatible avec une durée. En se composant avec les opérateurs aspectuels du modus, les schèmes associés aux unités lexicales sont intégrés dans des constructions prédicatives aspectualisées déterminées par les opérations de prise en charge de l'acte d'énonciation. Ainsi, le schème lexical statique associé à 'être honnête' peut être présenté dans un énoncé sous la forme d'un processus en cours (Luc devient honnête) tandis que le schème lexical événementiel associé à 'se garer' peut être verbalisé sous la forme d'un processus actuel (Luc est en train de se garer dans le parking du grand magasin) ou d'un processus passé (Luc se garait lorsqu'un camion s'est arrêté empêchant toute manœuvre) ou encore d'un état résultant (Enfin Luc a bien garé sa voiture, il est maintenant tranquille)... Nous renvoyons à d'autres publications pour l'analyse et la représentation détaillées de ces problèmes.

\section{Références bibliographiques}

Asher, N. 2011. A snapshot of discourse semantics in 2011. Mémoires de la Société Linguistique de Paris, Nouvelle série, t. XX, L'architecture, les modules et leurs interfaces, 183-203. LeuvenParis : Peeters.

Bally, Ch. 1932/1965. Linguistique générale et linguistique française. Berne : Franke.

Benveniste, E. 1966. Problèmes de linguistique générale, Vol. I. Paris : Gallimard.

Benveniste, E. 1974. Problèmes de linguistique générale, Vol. II. Paris : Gallimard.

Church, A. 1940. A formalization of the Simple Theory of Types, Journal of Symbolic Logic, 5: 5668.

Church, A. 1941. The Calculi of Lambda-Conversion. Annals of Mathematics Studies, Vol. 6. Princeton: Princeton University Press.

Comrie, B. 1981. Language Universals and Linguistic Typology. Chicago: Chicago University Press. 
Culioli, A. 1990. Pour une linguistique de l'énonciation, Vol. I. Paris : Ophrys.

Culioli, A. 1999. Pour une linguistique de l'énonciation, Vol. IV. Paris : Ophrys.

Culioli, A. 2002. Variations sur la linguistique. Paris: Klincksieck.

Curry, H., Feys, R. \& Craig, W. 1958. Combinatory Logic. Amsterdam: North Holland Publishing Company.

Curry, H., Hindley, R. \& Seldin, J. 1972. Combinatory Logic, vol. II. Amsterdam: North Holland Publishing Company.

Desclés, J.-P. 1987. Réseaux sémantiques : la nature logique et linguistique des relateurs. Langages, $87: 55-78$.

Desclés, J.-P. 1990. Langages applicatifs, langues naturelles et cognition. Paris : Hermès.

Desclés, J.-P. 2001. Prépositions spatiales, relateurs et préverbes, Etudes cognitives 4, 13-30. Warszawa: Académie des Sciences de Pologne, Institut de slavistique.

Desclés, J.-P. 2004. Analyse syntaxique et cognitive des relations entre la préposition sur et le préverbe sur-en français, Etudes cognitives 6, 21-46. Warszawa: Académie des Sciences de Pologne, Institut de slavistique.

Desclés, J.-P. 2005. La polysémie verbale. Un exemple : le verbe avancer. In O. Soutet (ed.), La Polysémie, 111-136. Paris : Presses de l'Université Paris-Sorbonne.

Desclés, J.-P. 2011a. Le problème de la polysémie verbale en français : donner en français, Cahiers de lexicologie, $\mathrm{N}^{\circ} 98: 95-111$.

Desclés, J.-P. 2011b. Une articulation entre syntaxe et sémantique cognitive: la Grammaire Applicative et Cognitive, Mémoires de la Société Linguistique de Paris, Nouvelle série, tome XX, L'architecture, les modules et leurs interfaces, 115-153. Leuven/Paris : Peeters.

Desclés, J.-P. 2016. A cognitive and conceptual approach to tense and aspect markers. In Zl. Guentchéva (ed.), Aspectuality and Temporality. Descriptive and theoretical issues, 27-60. Amsterdam/Philadelphia: John Benjamins Publishing Company.

Desclés J.-P. et Biskri I. 1997, Applicative and Combinatory Categorial Grammar (from syntax to

functional semantics). In R. Mitkov \& N. Nicolov (eds.), Recent Advances in Natural Languages

Processing, 71-84. Amsterdam: John Benjamins Publishing Company.

Desclés, J.-P. et Guentchéva, Z. 1995. Is notion of Process necessary? In P. M. Bertinetto et al. (eds) Temporal reference, Aspects and Actionality. Semantic and Syntactic Perspectives, Vol I., 55-70. Torino: Rozenberg \& Sellier.

Desclés, J.-P. et Guentchéva, Z. 1998. Causality, causativity, transitivity. In L. Kulikov \& H. Vater, Typology of verbal Categories, Papers Presented to Vladimir Nedjalkov on the Occasion of his 70th Birthday, 7-27. Tübingen: Niemeyer.

Desclés, J.-P. et Guentchéva, Z. 2012. Universals and Typology. In R. Binnick (ed.), Oxford Handbook of Tense and Aspect, 123-154. New York: Oxford University Press.

Desclés, J.-P., Guibert G. et Sauzay, B. 2016. Logique combinatoire et Lambda-calcul : des logiques d'opérateurs, Vol. I ; Calculs des significations par une logique d'opérateurs, Vol. II : * Vers une logique d'opérateurs; ** Concepts et schèmes analysés par la logique combinatoire. Toulouse : Cépaduès.

Dhombres, J. 1978, Nombre, mesure et continu. Épistémologie et histoire. Paris : CEDIC/Fernand Nathan.

Dowty, D. 1979. Word Meaning and Montague Grammar. Dordrecht/Boston/London: D. Reidel Publishing Company.

François, J. 2007. Pour une cartographie de la polysémie verbale. Leuven-Paris : Peeters.

Fuchs, C. et B. Victorri. 1996. La polysémie, construction dynamique du sens. Paris : Hermès.

Garcia, D. 1998. COATIS, un système de traitement automatique qui localise les expressions d'actions reliées causalement dans les textes, Thèse de doctorat, Université Paris-Sorbonne.

Guillaume, G. 1969. Langage et science du langage. Paris : Nizer.

Hagège, Cl. 1982. La structure des langues. Paris : Presses Universitaires de France.

Harris, Z. 1976. Notes du cours de syntaxe. Paris : Edition du Seuil.

Jackiewicz, A. 1999. Causalité et prise en charge énonciative. Etudes Cognitives, vol. 3 : 249-270. Warszawa : Académie des Sciences de Pologne, Institut de slavistique.

Kant, E. 1787[2012]. Critique de la raison pure. Paris : Presses Universitaires de France.

Kleiber, G. 1999. Problèmes de sémantique. La polysémie en question. Villeneuve: Presses Universitaires du Septentrion.

Langacker, R. 1987. Foundations of Cognitive Grammar, Vol. I, Theoretical Prerequisites. Stanford: Stanford University Press. 
Langacker, R. 1991. Foundations of Cognitive Grammar, Vol. II. Stanford: Stanford University Press.

Lewicka, H., Bogacki K. et al. 1983. Dictionnaire sémantique et syntaxique des verbes français. Warszawa : Panstwowe Wydawnictwo Naukowe.

McCawley, J. 1968. The Role of Semantics in a Grammar. In E. Bach \& P. Harms (eds.) Universals in Linguistic Theory, 124-169. New York: Holt, Rinehart \& Winston.

Martin, R. 2016. Linguistique de l'universel. Réflexions sur les universaux du langage, les concepts universels, la notion de langue universelle. Paris : Académie des inscriptions et belles-lettres.

Picoche, J. 1986. Structures sémantiques du français. Paris : Nathan.

Picoche, J. et Rolland, J.-C. 2002. Dictionnaire du français usuel. Bruxelles: De Boeck Duculot.

Pottier, B. 2000. Représentations mentales et catégorisations linguistiques. Louvain-Paris : Editions Peeters.

Pottier, B. 2012. Images et modèles en linguistique. Paris : Honoré Champion.

Seiler, H. \& , G. Brettschneider (eds.). 1985. Language Invariants and Mental Operations. Tübingen: Narr.

Hindley R. \& Seldin, J. 2010. Lambda-calculus and Combinators, an Introduction. Cambridge: Cambridge University Press.

Reichenbach, H. 1947 [1966]. Elements of Symbolic Logic. New York: The Free Press.

Shaumyan, S. 1987. A semiotic Theory of Natural Langue. Bloomington: Indiana University Press.

Soutet, O. (ed.). 2005. La Polysémie. Paris: Presses de l'Université Paris-Sorbonne.

Talmy, Leonard. 1988. The relation of Grammar to Cognition. In B. Rudzka-Ostyn (ed.), Topics in Cognitive Linguistics, 165-205. Amsterdam: John Benjamins Publishing Company.

Thom, R. 1981. Modèles mathématiques de la morphogénèse. Paris : Christian Bourgois.

Thom, R. 1983. Paraboles et catastrophes. Paris : Flammarion.

Wittgenstein, L. 1922[1993]. Tractatus logico-philosophicus, trad. de l'allemand par Gilles Gaston Granger. Paris : Gallimard.

\section{Notes de fin}

\footnotetext{
* Nous remercions les deux relecteurs anonymes pour leurs remarques et critiques de la précédente version de cet article. Nos remerciements vont également à Raphaëlle Chossenot pour la relecture attentive de la dernière version de cet article.

${ }^{\mathrm{i}}$ Corresponding author : guentche@vjf.cnrs

${ }^{\text {ii }}$ Sur ce point, notre analyse diffère de nombreuses autres analyses, par ex., de celles de McCawley (1968) et de Dowty (1979) dans la représentation de 'to kill' où l'agentivité et la causalité ne sont pas distinguées.

${ }^{\text {iii }}$ Sur ce point voir aussi Seiler \& Brettschneider (1985) et Martin (2016).

${ }^{\text {iv }}$ Les $\lambda$-expressions et le $\lambda$-calcul ont été introduits par Church $(1940,1941)$, utilisé en sémantique formelle (voir par exemple Asher (2011) dans la SDRT). Sur une introduction au $\lambda$-calcul, voir Desclés et al. (2016).

${ }^{v}$ Voir aussi Desclés et al. (2016).

${ }^{\mathrm{vi}}$ Sur la notion de contrôle, voir Comrie (1981), Hagège (1982).

vii Les primitives 'FAIRE' et 'CONTR' ne sont pas des "primitives de causalité » puisqu'elles construisent une situation dynamique, alors que la causalité relie deux situations, l'une étant la cause de l'autre (comme La venue de Pierre a entraîné le départ de sa sœur Marie), d'où la primitive CAUSE et ses différentes spécifications ( «augmenter », " diminuer », «éliminer » ...). Sur ce point, voir Garcia (1998), Jackewicz (1999) et Desclés et Guentchéva (1998).

viii L'acte d'énonciation analysé comme un processus inaccompli (Desclés 2016) est une position théorique qui nous éloigne du modèle de Reichenbach (1947/1966) et des approches qui s'y réfèrent plus ou moins directement.

${ }_{i x}$ En bulgare, par exemple, l'état résultant est rendu par le Parfait et l'événement par l'Aoriste.

${ }^{\mathrm{x}}$ La continuité a réellement été correctement appréhendée sur le plan philosophique et définie à la fin du XIX ${ }^{\text {ième }}$ siècle seulement par les mathématiciens Cauchy et Dedekind (voir Dhombres 1978 : 199246). Auparavant, la continuité était assimilée à la notion de densité (entre deux points, ou instants, il existe toujours un point, ou instant, intermédiaire). Pour Dedekind, une coupure d'un intervalle
} 
continu entre la partie A antérieure à $\mathrm{B}$, introduit (i) soit un dernier instant dans A (une borne fermée terminale de A) mais pas un premier instant dans $\mathrm{B}$ (une borne ouverte de $\mathrm{B}$ ), (ii) soit une absence de dernier instant dans A (une borne ouverte de A) mais un premier instant dans B (une borne fermée initiale de B). La coupure continue entre A et B exclut donc la possibilité d'avoir un saut entre un dernier instant de $\mathrm{A}$ et un premier instant de $\mathrm{B}$ ou encore la possibilité d'avoir un élément (une lacune) entre $\mathrm{A}$ et $\mathrm{B}$ qui ne serait ni dans $\mathrm{A}$ ni dans $\mathrm{B}$, comme «la racine de 2 » (un nombre irrationnel) est une lacune dans la classe des nombres rationnels (qui n'est pas continue), bien que ce nombre irrationnel puisse être approché, aussi près que l'on veut, par une suite croissante $A_{n}$ et une suite décroissante $B_{n}$ de nombres rationnels.

${ }^{x i}$ Dans ces exemples, la présence de la préposition sur signifie que 'passage-piétons' dénote un lieu qui possède une surface par rapport à laquelle la voiture peut être repérée. 